\title{
Assisted Reproduction Technologies Impair Placental Steroid Metabolism
}

\author{
Abby C. Collier ${ }^{2}$, Shogo J. Miyagi ${ }^{2}$, Yasuhiro Yamauchi ${ }^{1}$, and Monika A. Ward ${ }^{1}{ }^{*}$ \\ 1 Institute for Biogenesis Research, Department of Anatomy, Biochemistry and Physiology, John A. Burns \\ School of Medicine, University of Hawaii, 1960 East-West Rd, Honolulu, Hawaii, 96822, USA \\ 2Department of Tropical Medicine, Medical Microbiology and Pharmacology, John A. Burns School of \\ Medicine, University of Hawaii, 651 Ilalo St, Honolulu, Hawaii, 96822, USA
}

\section{Abstract}

The placenta plays a vital role in pregnancy by facilitating steroid passage from maternal to fetal circulation and/or direct production of hormones. Using a murine model, we demonstrated the differences in placental steroid metabolism between pregnancies conceived naturally and with assisted reproduction technologies (ART): in vitro fertilization (IVF) and intracytoplasmic sperm injection (ICSI). While the ovarian steroid production was similar (estrone, 17/-estradiol) or higher (estriol) in ART pregnancies compared to mating, the levels of placental estriol were significantly lower in ART group. Placentas from ART had significantly higher activities of the steroid metabolizing enzymes UDP-glucuronosyltransferase (UGT) and sulfotransferase (SULT), which in ICSI were also coupled with decreased activity of the steroid regenerating enzymes $\beta$-glucuronidase $(\beta-G)$ and Aryl sulfatase (AS). Levels of steroid metabolites androstane-3 $\alpha$-17 $\beta$-diol glucuronide and dehydroepiandrosterone sulfate were higher in fetal compared to maternal blood in ART, but not in mating. This study demonstrates that in murine ART pregnancies, higher metabolism and clearance of steroids by the placenta may seriously affect the passage of essential hormones to the fetus. If a similar phenomenon exists in humans, this could provide a plausible explanation for obstetric and neonatal complications associated with ART, including the higher incidence of low birth weight babies.

\section{Keywords}

ART; IVF; ICSI; Placenta; Steroids

\section{Introduction}

In the latter half of the twentieth century declining fertility has become a major problem in developed countries. Although the causes and solutions for infertility are controversial, it is well documented that each year more and more couples are turning to assisted reproduction technologies (ART) in order to conceive [1]. The increasing use of ART has allowed thousands of infertile couples to have children, accounting for $1 \%$ of all births and $18 \%$ of multiple births

\footnotetext{
*Correspondence: Monika A Ward, Institute for Biogenesis Research, Department of Anatomy, Biochemistry and Physiology, John A. Burns School of Medicine, University of Hawaii, 1960 East-West Rd, Honolulu, HI, 96822. Phone: 808-956-0779, Fax: 808-956-7316, E-mail: E-mail: mward@ hawaii.edu.

Publisher's Disclaimer: This is a PDF file of an unedited manuscript that has been accepted for publication. As a service to our customers we are providing this early version of the manuscript. The manuscript will undergo copyediting, typesetting, and review of the resulting proof before it is published in its final citable form. Please note that during the production process errors may be discovered which could affect the content, and all legal disclaimers that apply to the journal pertain.
} 
in the United States [2]. Although the majority of ART children are normal, there are concerns about the risk of adverse pregnancy outcomes [3]. Pregnancies achieved by in vitro fertilization (IVF) and intracytoplasmic sperm injection (ICSI) require a greater number of obstetric interventions such as induced labor and caesarean section, and are also at higher risk of obstetric and perinatal complications, including premature births, small-for-gestational age babies and congenital abnormalities [3-7]. It is presently unclear if these increased risks are attributable to the underlying causes of infertility, characteristics of the infertile couple, or the use of ART.

Abnormal placental characteristics have been reported with ART pregnancies including higher rates of placenta previa, placental abruption, premature rupture of the membranes and preeclampsia [3,6,8]. Pathologic abnormalities of ART placentas have also been described, including abnormal placental shape and abnormal umbilical cord insertion in humans, and higher placental weights in mice [9-11]. Despite its vital importance, the role of the placenta in complications of pregnancy and neonatal or pediatric outcomes is often underestimated. With the exception of pre-term labor, researchers seldom consider the likelihood that functional placental impairment is pivotal in obstetric and developmental abnormalities.

Given the importance of the placenta throughout pregnancy, and the evidence of placental abnormalities in ART pregnancies, we hypothesized that ART may affect placental steroid metabolism and that this may be a cause or contributory factor in obstetric and neonatal complications associated with assisted reproduction. While much is known about the production of steroids and their role during pregnancy for humans and other species, the activity and importance of placental steroid metabolism and elimination remains unclear. Steroid metabolism during pregnancy is different between humans and other species. In humans, steroid production is dependent on the coordinate action of the placenta, fetal liver and fetal adrenal. In contrast, in rodents, the majority of steroids are produced in the ovaries and they are continuously delivered to the fetuses through the placenta. Regardless of the different origins for steroid production, the role of the placenta in steroid metabolism and elimination is vital in both human and rodent species.

Here we investigated the activity of steroid clearance and regeneration enzymes in placentas from mice that were impregnated by normal mating, IVF or ICSI. We also assessed specific clearance of steroids by comparing the levels of terminal glucuronide and sulfate metabolites in maternal and fetal blood. Finally, we determined the efficiency of the fetal-placental unit in producing steroids by detection of estriol levels in maternal blood. Rather than focusing on steroid production as many other researchers have done, we examined placental steroid conjugation and elimination to assess the role of the placental metabolism in maintaining pregnancy and proper fetal development. The results of our study indicate that there are significant differences in placental steroid metabolism between ART (especially ICSI) and normal fertilization pregnancies.

\section{Materials and methods}

\subsection{Reagents}

Mineral oil was purchased from Squibb and Sons (Princeton, NJ); pregnant mares' serum gonadotrophin (eCG), human chorionic gonadotrophin (hCG), and Dehydroepiandrosterone Sulfate (DHEAS) ELISA tests were purchased from Calbiochem (Spring Valley, CA); ELISA tests for $5 \alpha$ androstane-3 $\alpha$-17 $\beta$-diol Glucuronide ( $3 \alpha$ diolG) were purchased from DRG International Inc. (Mountainside, NJ); 3'-phosphoadenosine-5' -phosphosulfate (PAPS) and 4nitrophenyl sulfate were purchased from EMD Biosciences (San Diego, CA); Estriol ELISA tests were purchased from Cayman Chemical Company (Ann Arbor, MI); 4methylumbelliferone glucuronide was purchased from Acros Organics (Geel, Belgium). All 
other chemicals were obtained from Sigma Chemical Co. (St Louis, MO), unless otherwise stated.

\subsection{Animals}

Mice B6D2F1 (C57BL/6 $\times$ DBA/2) and CD-1 were obtained at 6 weeks of age from National Cancer Institute (Raleigh, NC) and from Charles River (Wilmington, MA), respectively. Mice B6D2F1 were used as sperm and oocytes donors and for mating, while CD-1 mice were used as surrogate mothers and vasectomized males for ART. The mice were fed ad libitum with a standard diet and maintained in a temperature and light-controlled room $\left(22^{\circ} \mathrm{C}, 14 \mathrm{~h} \mathrm{light} / 10\right.$ $\mathrm{h}$ dark), in accordance with the guidelines of the Laboratory Animal Services at the University of Hawaii and guidelines presented in National Research Council's (NCR) "Guide for Care and Use of Laboratory Animals" published by Institute for Laboratory Animal Research (ILAR) of the National Academy of Science, Bethesda, MD, 1996. The protocol for animal handling and treatment procedures was reviewed and approved by the Animal Care and Use Committee at the University of Hawaii.

\subsection{In Vitro Fertilization (IVF)}

The method for sperm capacitation and IVF using T6 medium [12] has been described by us previously [13]. The oocytes were obtained from 8-12 weeks old females induced to superovulate with injections of $5 \mathrm{iu}$ eCG and 5iu hCG given $48 \mathrm{~h}$ apart. Oviducts were removed 14-15 $\mathrm{h}$ after the injection of hCG. Epididymal spermatozoa were obtained from males 8-16 weeks of age and were capacitated in T6 medium for $1.5 \mathrm{~h}$ at $37^{\circ} \mathrm{C}$ in a humidified atmosphere of $5 \% \mathrm{CO}_{2}$ in air prior to fertilization. Fertilization took place in $200 \mu \mathrm{L}$ drops of T6 medium containing the contents of four oviducts, and capacitated sperm at final concentration of $\sim 2 \times$ $10^{6} / \mathrm{mL}$. Gametes were co-incubated for $4 \mathrm{~h}$, after which the oocytes were washed several times with HEPES-CZB medium [14], followed by at least one wash with CZB medium [15]. Only morphologically normal oocytes were selected for culture and transfer.

\subsection{Intracytoplasmic Sperm Injection (ICSI)}

The oocytes were obtained as for IVF except that cumulus-oocyte complexes were released from the oviducts into $0.1 \%$ of bovine testicular hyaluronidase (300 USP units $/ \mathrm{mg}$ ) in HEPESCZB medium to disperse cumulus cells. The cumulus-free oocytes were washed with HEPESCZB medium and used immediately for ICSI. Epididymal spermatozoa were obtained from males 8-16 weeks of age, dispersed in $0.5 \mathrm{~mL}$ of HEPES-CZB medium, and incubated briefly at room temperature. Highly motile sperm from the top of the suspension were taken for injections. ICSI was carried out as described by us recently [16]. Briefly, a small drop of sperm suspension was mixed thoroughly with an equal volume of HEPES-CZB containing 12\% (w/ v) polyvinyl pyrrolidone (PVP, $\mathrm{M}_{\mathrm{r}} 360 \mathrm{kDa}$ ) immediately before ICSI. Injections were performed using Eppendorf Micromanipulators (Micromanipulator TransferMan, Eppendorf, Germany) with a Piezo-electric actuator (PMM Controller, model PMAS-CT150, Prime Tech, Tsukuba, Japan). Sperm heads and tails were separated by applying one or more piezo pulses and only heads were injected. Injections were done in HEPES-CZB within $1 \mathrm{~h}$ after oocyte collection and only motile sperm were chosen for injections. Sperm-injected oocytes were transferred into CZB medium for culture. The oocytes were examined at $\sim 6 \mathrm{~h}$ after ICSI to assess their survival and activation. The oocytes with two well-developed pronuclei and the distinct $2^{\text {nd }}$ polar body were recorded activated. They were then taken for embryo culture and transfer.

\subsection{Embryo Culture and Transfer}

After IVF and ICSI, the oocytes were placed in $50 \mu \mathrm{L}$ drops of CZB medium pre-equilibrated overnight with humidified $5 \% \mathrm{CO}_{2}$ in air. The culture drops were contained in plastic culture 
dishes (Falcon, Bedford, MA) and overlaid with mineral oil. The number of 2-cell embryos was recorded after $24 \mathrm{~h}$ in culture. Embryos at the 2-cell stage were transferred to the oviducts (14 to 16 per oviduct) of CD-1 females mated during the previous night with vasectomized CD-1 males. On day $18^{\text {th }}$ of gestation, caesarian section was performed, and placentas and fetuses were harvested.

\subsection{Tissue Collection}

Placentas and ovaries were washed briefly in Dulbecco PBS, drained, and placed singly into $1 \mathrm{~mL}$ tubes. Fetuses were sacrificed by decapitation and whole blood was collected into 0.5 $\mathrm{mL}$ tubes containing minute amount of heparin. Whole blood was also collected from dams through cardiac puncture under anesthesia immediately prior to caesarian section. Plasma was separated by centrifugation $\left(7,000 \times g, 5 \mathrm{~min}, 25^{\circ} \mathrm{C}\right)$ and transferred to new tubes. During collection all tissue samples were kept on ice for up to $30 \mathrm{~min}$ prior to freezing. Samples were frozen at $-80{ }^{\circ} \mathrm{C}$ until use.

\subsection{Tissue Processing}

Placentas and ovaries were thawed, wet weight recorded, and homogenized 1:4 in Tris- $\mathrm{HCl}$ buffer containing $5 \mathrm{mM} \mathrm{MgCl}_{2}$ (pH 7.4). An aliquot of homogenate was reserved and frozen at $-80^{\circ} \mathrm{C}$ until use. Homogenates were subsequently centrifuged at $20,000 \times g$ to remove nuclei, mitochondria and cellular cytoskeleton, then the supernatant centrifuged at $100,000 \times g$ to produce microsomal and cytosolic fractions. These fractions were frozen at $-80^{\circ} \mathrm{C}$ until use.

\subsection{Biochemical Assays for Steroid Metabolizing Enzymes}

Four enzymes involved in steroid clearance in placentas were tested: glucuronosyltransferase (UGT), sulfotransferase (SULT), $\beta$-glucuronidase ( $\beta-G$ ), and aryl sulfatase C (AS) (Table 1). All placental fractions were normalized to $2 \mathrm{mg} / \mathrm{mL}$ protein using the Bicinchoninic acid method [17]. Activities of UGT, $\beta-G$, and AS were measured in placental microsomes, and activity of SULT was measured in placental cytosol. Total UGT activity was assessed with the substrate 4-methylumbelliferone as previously described [18] except that alamethicin $(0.5 \mu \mathrm{g} /$ $\mathrm{mg}$ protein) was used as the activator. The activity of $\beta-\mathrm{G}$ was measured using the substrate 4-methylumbelliferone glucuronide according to the method of Trubetskoy and Shaw [19]. Total SULT activity was measured using the $400 \mu \mathrm{M}$ 4-nitrophenol which at this level detects the following SULT family members: SULT1A1 (both the $* 1$ and $* 2$ alleles), 1A3 and 1E1 activity with a modest contribution from SULT2A1, as previously described [20,21]. The activity of AS was measured with 4-nitrophenyl sulfate [22]. The activity rates were derived by comparisons to standard curves of 4-methylumbelliferone (UGT, $\beta-G$ ) and 4-nitrophenol (SULT and AS). Validation parameters for the UGT and $\beta-G$ assays were: standard curve $r^{2}$ $=0.9981$, slope $=494.5 \pm 12(\mathrm{CV} 2.5 \%)$ with a limit of detection of $0.1 \mu \mathrm{M}$, defined as the minimum concentration on the standard curve statistically different from background. Validation parameters for the SULT and AS assays were: standard curve $\mathrm{r}^{2}=0.9992$, slope $=$ $0.003492 \pm 2.4 \times 10^{-5}$ (CV $0.66 \%$ ) with the limit of detection of $1 \mu \mathrm{M}$.

\subsection{ELISA Assays for Metabolized Steroids}

Two terminal metabolites, androstane-3 $\alpha-17 \beta$-diol glucuronide ( $3 \alpha$ diolG) and sulfated steroid dehydroepiandrosterone sulfate (DHEAS) were tested using blood plasma from dams and fetuses. Additionally, the levels of the unconjugated steroids estrone, 17ßestradiol and estriol were measured in placental tissue (Table 1) and in the ovaries from mated, IVF and ICSI dams. The ELISAs were performed according to manufacturer's instructions and all measurements were performed in duplicate. Plasma from dams was measured individually for each of 3 mated, IVF and ICSI dams. Fetal blood plasma was pooled from fetuses belonging to each dam (Mated: $\mathrm{n}=7, \mathrm{n}=9, \mathrm{n}=11$; IVF: $\mathrm{n}=15, \mathrm{n}=7$; ICSI: $\mathrm{n}=11, \mathrm{n}=12, \mathrm{n}=7$ ). The appropriateness of 
pooling blood was assessed statistically using Dunnett's multiple comparison test which showed no significant differences between pools of blood from each dam for either $3 \alpha$ diolG or DHEAS. Using Dunnett's multiple comparison in this way checks for effects that may be common to all fetuses from a single dam. Because there were no significant differences between groups, we can infer that the pooling was appropriate since the influence of dam specific variables on fetus levels of metabolites was negligible. For steroid level measurements ovaries and placentas were homogenized as described earlier, homogenates from placentas originating from fetuses belonging to each dam were pooled (Mated: $n=9, n=10$ and $n=11$;VF: $n=$ $8, n=15$ and $n=7$; ICSI: $n=11, n=12$ and $n=7$ ) while homogenates of maternal ovaries were assessed individually with $n=3$ ovaries from each of normally mated, IVF and ICSI dams.

\subsection{Statistical Analyses}

Statistical analyses were performed using Prism 3.0 with statistical significance set at $\alpha=0.05$. (GraphPad Prism, San Diego, CA). Parametric statistics were performed since all data approximated Gaussian distributions, and two-tailed student $t$ tests were used to assess differences between groups. When paired sample $t$-tests were performed, the correlation coefficient was generated to assess the effectiveness of the pairing. It is noted that in the case of pooled fetal blood analyses, only $n=2$ pools were used for IVF fetuses hence although a standard deviation for these data can be derived and comparisons made, statistical inferences may not be robust. Multiple regression analysis was performed on abortion sites and successful pregnancies to determine the effects of the ICSI procedure.

\section{Results}

\subsection{Production of Fetuses after Mating, IVF and ICSI}

Fetuses were obtained after mating, IVF and ICSI (Table 2). Three females were mated and all of them became pregnant providing a total of 30 fetuses. When embryos produced by IVF and ICSI were transferred into the oviducts of pseudopregnant females, all females ( 3 per group) became pregnant, and similarly to mating, 30 fetuses were obtained in each group. The occurrence of 30 fetuses/placentas in each group was purely by chance and no fetuses/placentas were selectively excluded. No abortion sites were noted after caesarian section in females that became pregnant after mating while abortion sites were present in both IVF and ICSI pregnancies. Multiple regression analysis indicated that the number of abortions per mouse was significantly affected by ART $(\mathrm{P}<0.01)$.

\subsection{Placental Weights}

Mean placental weights were significantly higher in both IVF $(114.2 \pm 21.2 \mathrm{mg}, \mathrm{n}=30$, mean $\pm \mathrm{SD})$ and ICSI $(105.5 \pm 17.5 \mathrm{mg}, \mathrm{n}=30)$ mice compared to normally mated mice $(84.3 \pm 15.8$ $\mathrm{mg}, \mathrm{n}=30, \mathrm{P}<0.001$ each, Fig. 1). There was no statistically significant difference in mean placental weight between ICSI and IVF groups $(\mathrm{P}=0.084)$.

\subsection{Activity of Steroid Metabolizing Enzymes}

The activity of steroid metabolizing enzymes was measured individually in 30 placentas from each group (Mated, IVF, and ICSI). The activity of UGT was observed in all placentas tested. Placentas from IVF pregnancies showed a small, but statistically significant $(\mathrm{P}<0.03$, Fig. 2A) increase in UGT activity, while those from ICSI pregnancies had highly elevated UGT (P $<0.001$, Fig. 2A). Analysis of $\beta-G$ activity showed no difference between placentas from IVF and Mated groups $(P=0.18)$ but a highly significant decrease in $\beta-G$ was observed in ICSI placentas compared to placentas from mated females $(\mathrm{P}<0.001$, Fig. 2B). The UGT rates were significantly higher than $\beta-\mathrm{G}$ activities in all tested groups $(\mathrm{P}<0.001)$. The systemic clearance 
of steroids by UGT performed by the placenta was 81 and 85 -fold greater for placentas from mated females and IVF females, respectively, than cleavage and recirculation by $\beta-G$.

However, ICSI placentas showed a substantial increase in placental clearance by glucuronidation. The rates of UGT in ICSI placentas averaged $14.9 \pm 4.1 \mathrm{nmol} / \mathrm{min} / \mathrm{mg}$ protein while $\beta-G$ activities averaged only $0.056 \pm 25 \mathrm{nmol} / \mathrm{min} / \mathrm{mg}$ protein; these data correspond to 266 -fold higher UGT activities than $\beta$-G, which equals 3 -fold greater systemic clearance by glucuronidation than observed for placentas from mated or IVF females $(\mathrm{P}<0.001)$.

In contrast to UGT, SULT activity was not observed in all placentas. Interestingly, more placentas from ART groups (IVF: 93\%, 28/30; ICSI: 97\%, 29/30) had measurable SULT activity compared to placentas from mated females $(67 \%, 20 / 30)$. These data correlate well with the greater levels of SULT activity observed in ART vs. Mated groups $(\mathrm{P}=0.04$ and $\mathrm{P}<$ 0.001 for IVF and ICSI, respectively, Fig. 3). The activity of AS was more frequently observed in IVF placentas $(70 \%, 21 / 30)$ compared to ICSI and Mated groups (60\%, 18/30, both groups), and this correlated with the higher AS activity rates. However, the difference between Mated vs. IVF and ICSI groups did not reach statistical significance (Mated vs. IVF: $\mathrm{P}=0.06$, Mated vs. ICSI: $\mathrm{P}=0.49$, Fig. 3). The rates, at which the opposing SULT and AS activities occurred in placentas from mated and IVF females, were similar. Therefore, placental clearance of steroids and chemicals by sulfonation did not significantly outweigh systemic recirculation and conservation of these molecules in placentas from mated and IVF females $(P=0.30$ and $\mathrm{P}=0.57$, respectively). In stark contrast, ICSI placentas had 4-fold greater sulfonation than recirculation $(2.2 \pm 1.4 \mathrm{nmol} / \mathrm{min} / \mathrm{mg}$ protein for SULT vs. $0.56 \pm 1.2 \mathrm{nmol} / \mathrm{min} / \mathrm{mg}$ protein for AS, P < 0.001). While the error bars appear large, because of the large number of samples tested (30 individual placentas in each group), significance was reached.

\subsection{Concentrations of Steroid Metabolites in Maternal and Fetal Blood}

We next measured the levels of terminal glucuronide (3adiolG) and sulfate (DHEAS) metabolites that reflect the specific clearance of steroids (i.e. are inversely proportional to the level of free steroid available). There were no significant differences in the levels of $3 \alpha$ diolG in maternal blood regardless of the type of pregnancy (Mated, IVF, and ICSI). Although there was a slight increase in the $3 a d i o l G$ levels in the blood of pups compared to their dams, this difference was not statistically significant $(\mathrm{P}=0.06$, Fig. $4 \mathrm{~A})$. This was not the case for IVF and ICSI fetuses that had more than two-fold higher levels of $3 \alpha$ diolG $(P=0.02$ and $\mathrm{P}=0.01$ respectively) than their dams (Fig. 4A). Furthermore, the fetuses conceived by ART had far higher levels of 3 diolG metabolites in their blood than the pups conceived by normal mating, that approached significance with IVF pups $(P=0.07)$, and was significant for ICSI fetuses ( $P$ $=0.05$ ). The levels of DHEAS were significantly higher in the fetal blood than the maternal blood in IVF and ICSI pregnancies $(\mathrm{P}=0.03$, paired $t$-test, Fig. 4B) but not in pregnancies originating from mating $(\mathrm{P}=0.4$, paired $t$-test, Fig. 4B)

\subsection{Levels of Estriol in Placentas}

We then measured the level of estriol which reflects the efficiency of total steroid production (being the end product of a biochemical pathway). Lower levels of estriol would be expected if steroids higher up the cascade are functionally cleared by glucuronidation and sulfonation. The level of estriol in placentas from IVF and ICSI groups was almost $80 \%$ and $70 \%$ lower, respectively, as compared with placentas from mated females (Fig. 5). This difference approached, but did not reach significance $(\mathrm{P}=0.098$ and $\mathrm{P}=0.091$ for IVF and ICSI, respectively) which is almost certainly due to the small sample size (i.e. using 3 pools for each condition); and would probably reach significance with greater numbers of placental pools tested. 


\subsection{Levels of Estrone, 17 $\beta$-estradiol and Estriol in Maternal Ovaries}

Because in mice maternal ovaries are the main source of steroids for developing fetuses we compared the levels of Estrone, $17 \beta$-estradiol and Estriol in ovaries from females impregnated by Mating, IVF and ICSI. The levels of estrone and 17 $\beta$-estradiol in ovaries from ART group females were lower or similar compared to Mated group (Fig. 6A-B) while the levels of estriol were higher in ART than in Mating.

\section{Discussion}

Our study provides the first evidence of dysregulation of placental steroid metabolism in mouse pregnancies conceived through ART. If a similar impairment exists in humans, it may contribute to obstetric and perinatal complications associated with assisted reproduction.

The increase in steroid metabolism and clearance was more evident in ICSI than in IVF pregnancies. Intracytoplasmic sperm injection is crucially different than IVF and mating in that it bypasses several important steps that are required in normal fertilization. In spite of high success rates with ICSI, it is possible that the method induces some technique-specific effects that are yet to be discovered. Here, we did not observe significant differences in the outcome of mouse IVF and ICSI, measured by proportion of embryos that developed to term. This is in agreement with our past studies, in which we demonstrated that mouse embryos produced with these two methods had similar potential to develop in vitro [23] and in vivo [24], and that ICSI did not enhance infertile phenotype or confer risks when it was applied serially [13]. However, our recent analyses, also in the mouse, revealed that the dynamics of sperm chromatin remodeling were different after ICSI and IVF [25]. The results of the current study provide evidence for differences between ICSI and IVF at another level, that of placental function.

Increased placental weight and placental dysfunction have been suggested as potential causes of neonatal mortality in cloned livestock $[26,27]$ and mice $[28,29]$ and in vitro embryo culture or transfer of embryos to pseudopregnant recipient mothers also results in greater placental birth weight $[27,30,31]$. Furthermore, it has been shown that placental overgrowth and dysfunction reflects aberrant expression of imprinted genes regulating placentation, which may lead to incomplete communication between the placenta and embryo/fetus [29]. We observed increased placental weights in IVF and ICSI groups compared to normal mating. To our knowledge, this is the first evidence of increased placental weights in mouse ART other than those reported for cloning. Changes in gross placental size would be expected to affect all aspects of biochemical and xenobiotic metabolism which is a potential cause of smaller fetal weights consistently observed with ART [4-7]. We compared placentas from mice at gestational day 18 (and not earlier) because at this timepoint the placentas are fully mature but not functionally senescent as they might be if we had waited for natural birth on or around day 20. Additionally, caesarian section was used since this does not subject the tissue to the birthing process where inflammatory mediators and/or oxidative stress can change enzyme metabolism dynamics.

Steroids are vital for correct growth, development and body patterning of fetus [32,33]. Steroidogenesis and steroid clearance in humans are regulated by the placenta throughout pregnancy, with assistance from the fetal adrenals and liver after 8 weeks of gestation. In contrast, in mice steroids cannot be produced by the placenta which has only low levels of aromatase (CYP19) activity and production by the maternal ovaries is the main source of steroids for mouse fetuses. Since steroid hormones are highly lipophilic, they freely diffuse into cells to cause their effects. This underscores the importance of the placenta in the mouse model since steroid hormones must reach the fetal compartment by crossing the placenta. After steroids effects are complete, their action is terminated through metabolism by SULT and UGT enzymes that between them account for almost all steroid clearance. The SULT enzymes are 
present at high levels in both the fetal liver and placenta in humans and mice with hepatic activity reaching adult levels during the second trimester of pregnancy [34]. The importance of placental SULTS has been further demonstrated since pregnant mice with placental SULT1E1 gene knocked out have excessive circulating steroid hormones, placental thrombosis and recurrent fetal loss occurred [33]. Similar to the SULTs, UGT enzymes are present and active in both human and rodent placentas [35-38]. However, unlike SULTs, UGT enzymes are not active in the human liver until after birth, and may take several years to fully develop; this is believed to be a reason why some chemicals that are not harmful to rodents cause congenital birth defects in humans [39-41]. Because metabolites produced by SULT and UGT can be regenerated back to parent steroids by AS and $\beta-G$ enzymes in fetal livers and placentas, it is the balance between SULT/AS and UGT/ $\beta-G$ that determines circulating steroid levels and ultimately, to how much active steroid mouse fetuses are exposed [36,42].

We have shown here that placental metabolism of chemicals by UGT was higher than $\beta$-Gmediated recirculation in all tested groups. In placentas from mated and IVF mice UGT activities were 81- and 85-fold higher than $\beta$-G, respectively, while ICSI placentas showed 266 -fold higher UGT activity than $\beta$-G. This corresponds to 3 -fold higher placental elimination of steroids and chemicals through the UGT pathway in ICSI pregnancies compared to mating and IVF. In the sulfonation pathway, SULT detoxification and AS recirculation activities were balanced in mated and IVF females, while in ICSI placentas SULT activities were significantly higher than AS accounting for 4-fold greater clearance by sulfonation. Overall this metabolic profile indicates that in ICSI pregnancies the balance of metabolism shifts sharply towards steroid (and chemical) removal. The increased clearance of steroids from the fetal compartment was potentially up to $800 \%$ higher in ICSI placentas than in placentas from mated females through the combined effect of the increases in the sulfonation and glucuronidation pathways. These results are further strengthened by our independent assessment of estrogenic steroid production by the maternal ovaries. Females from ART groups had similar or lower levels of ovarian estrone and 17 $\beta$-estradiol but higher levels of estriol compared to females from Mated group. Our study also shows massive decrease in estriol levels in placental tissue as well as higher levels of steroid metabolites in fetal but not maternal serum in ART pregnancies. This, coupled with the finding that maternal ovaries in ART actually produce more estriol, provides strong evidence for the role of placental metabolism in removing steroids during transit to the fetal compartment.

An increase in steroid clearance in ART pregnancies is likely to have severe consequences. In humans, alteration in placental enzyme activity would potentially have more significant effects than in mice since the placenta is not merely a delivery vector for steroids (as is in the mouse) but the actual site of steroid synthesis. The relevance of our results, whilst occurring in a mouse model, are supported by recent findings from Zhang et al who demonstrated that proteins from ICSI placentas in humans differed from normally conceived placentas [43]. Differentially regulates proteins in the human placentas included proteins involved in membrane trafficking, metabolism, nucleic acid processing, stress response and cytoskeleton.

Although the passage of steroids between the maternal and fetal circulations via the placenta is diffusive, their metabolites (that are very hydrophilic) have poor ability to cross the placenta and other membranes. Hence the levels of metabolites in maternal and fetal blood are inversely reflective of steroid concentrations. Increased clearance and removal by both UGT and SULT pathways is expected to lower the levels of steroids and other chemicals in the fetal compartment and raise metabolite levels. The observed higher levels of both $3 \alpha \mathrm{diolG}$ and DHEAS metabolites in blood from ART fetuses compared to their dams support this. Lack of significant differences between metabolite levels in fetal and maternal blood in mating indicates that increased steroid clearance may be due to the changes in placental enzyme dynamics described. However, the exact cause of increased metabolites in fetal blood may be 
more complicated due to the contributions of fetal liver and/or kidney to metabolism and clearance. Since the fetus can only receive steroid from maternal blood (not conjugated glucuronides or sulfates are too polar to cross the placenta), the steroid levels in dams blood internally controls for the contribution of the maternal liver to this process. Finally, the lower levels of estriol produced ART placentas further supports our assertion of increased steroid clearance.

\section{Conclusions}

This is the first report showing that placentas from ART pregnancies have significantly altered steroid metabolism as compared with normal controls in mice. Low levels of steroid hormones delivered to the developing fetus would be expected to result in growth restriction, congenital malformations, genital abnormalities, and discordant changes in the endocrine axes. All of these syndromes have been reported with significantly elevated incidence in IVF and ICSI children [44-46]. Thus, if a similar dysregulation of steroid metabolism as that noted in mice occurs also in humans it may provide a plausible explanation for the neonatal, pediatric and adult diseases associated with assisted reproduction.

Based on our data from the mouse model we propose that adverse reproductive outcomes from ART may, at least in part, be due to induction of detoxification enzymes in placentas that is not paralleled by homeostatic increases in steroid recirculation enzymes. Our future work will involve testing the enzymes engaged in steroid metabolism in human placentas, and the corresponding metabolite profiles in fetal and maternal blood to gauge the corresponding human placental dynamics. The results of our work advance understanding of ART effects on placental steroid metabolism and clearance, and may assist in developing strategies to improve the efficiency and safety of assisted reproduction. Ultimately, these studies may assist in decreasing the levels of unsatisfactory obstetric, neonatal and pediatric outcomes when assisted reproduction in required.

\section{Acknowledgments}

This material is based on work supported by NIH HD048446, HD048845, and RR024206 (Project 2) grants to M.A.W. and NIH/NCRR 1P20RR024206-01A1 and UH-JABSOM start up funds to A.C.C.

\section{References}

1. US, D.o.H.a.H. Services. Assisted Reproduction Technologies Success Rates. National Summaries and Fertility clinic reports 2003. 2006

2. Wright VC, Schieve LA, Reynolds MA, Jeng G. Assisted reproductive technology surveillance--United States, 2000. MMWR Surveill Summ 2003;52:1-16. [PubMed: 14532867]

3. Reddy UM, Wapner RJ, Rebar RW, Tasca RJ. Infertility, assisted reproductive technology, and adverse pregnancy outcomes: executive summary of a National Institute of Child Health and Human Development workshop. Obstet Gynecol 2007;109:967-977. [PubMed: 17400861]

4. Allen VM, Wilson RD, Cheung A. Pregnancy outcomes after assisted reproductive technology. J Obstet Gynaecol Can 2006;28:220-250. [PubMed: 16650361]

5. Buckett WM, Chian RC, Holzer H, Dean N, Usher R, Tan SL. Obstetric outcomes and congenital abnormalities after in vitro maturation, in vitro fertilization, and intracytoplasmic sperm injection. Obstet Gynecol 2007;110:885-891. [PubMed: 17906024]

6. Kallen B, Finnstrom O, Nygren KG, Otterblad Olausson P, Wennerholm UB. In vitro fertilisation in Sweden: obstetric characteristics, maternal morbidity and mortality. Bjog 2005;112:1529-1535. [PubMed: 16225574]

7. Shevell T, Malone FD, Vidaver J, Porter TF, Luthy DA, Comstock CH, Hankins GD, Eddleman K, Dolan S, Dugoff L, Craigo S, Timor IE, Carr SR, Wolfe HM, Bianchi DW, D’Alton ME. Assisted 
reproductive technology and pregnancy outcome. Obstet Gynecol 2005;106:1039-1045. [PubMed: 16260523]

8. Romundstad LB, Romundstad PR, Sunde A, von During V, Skjaerven R, Vatten LJ. Increased risk of placenta previa in pregnancies following IVF/ICSI; a comparison of ART and non-ART pregnancies in the same mother. Hum Reprod 2006;21:2353-2358. [PubMed: 16728419]

9. Gavriil P, Jauniaux E, Leroy F. Pathologic examination of placentas from singleton and twin pregnancies obtained after in vitro fertilization and embryo transfer. Pediatr Pathol 1993;13:453-462. [PubMed: 8372030]

10. Jauniaux E, Englert Y, Vanesse M, Hiden M, Wilkin P. Pathologic features of placentas from singleton pregnancies obtained by in vitro fertilization and embryo transfer. Obstet Gynecol 1990;76:61-64. [PubMed: 2359567]

11. Van Thuan N, Wakayama S, Kishigami S, Ohta H, Hikichi T, Mizutani E, Bui HT, Wakayama T. Injection of somatic cell cytoplasm into oocytes before intracytoplasmic sperm injection impairs fullterm development and increases placental weight in mice. Biol Reprod 2006;74:865-873. [PubMed: 16436529]

12. Quinn P, Barros C, Whittingham DG. Preservation of hamster oocytes to assay the fertilizing capacity of human spermatozoa. J Reprod Fertil 1982;66:161-168. [PubMed: 7120180]

13. Ward MA. Intracytoplasmic sperm injection effects in infertile azh mutant mice. Biol Reprod 2005;73:193-200. [PubMed: 15800178]

14. Kimura Y, Yanagimachi R. Intracytoplasmic sperm injection in the mouse. Biol Reprod 1995;52:709720. [PubMed: 7779992]

15. Chatot CL, Ziomek CA, Bavister BD, Lewis JL, Torres I. An improved culture medium supports development of random-bred 1-cell mouse embryos in vitro. J Reprod Fertil 1989;86:679-688. [PubMed: 2760894]

16. Szczygiel, MA.; Yanagimachi, R. Intracytoplasmic Sperm Injection. In: Nagy, A.; Gertsenstein, M.; Vintersten, K.; Behringer, RR., editors. Manipulating the Mouse Embryo - A Laboratory Manual. Vol. 3. New York: Cold Spring Harbor Laboratory Press; 2003. p. 585-597.

17. Smith PK, Krohn RI, Hermanson GT, Mallia AK, Gartner FH, Provenzano MD, Fujimoto EK, Goeke $\mathrm{NM}$, Olson BJ, Klenk DC. Measurement of protein using bicinchoninic acid. Anal Biochem 1985;150:76-85. [PubMed: 3843705]

18. Collier AC, Tingle MD, Keelan JA, Paxton JW, Mitchell MD. A highly sensitive fluorescent microplate method for the determination of UDP-glucuronosyl transferase activity in tissues and placental cell lines. Drug Metab Dispos 2000;28:1184-1186. [PubMed: 10997937]

19. Trubetskoy OV, Shaw PM. A fluorescent assay amenable to measuring production of beta-Dglucuronides produced from recombinant UDP-glycosyl transferase enzymes. Drug Metab Dispos 1999;27:555-557. [PubMed: 10220481]

20. Mulder GJ, van Doorn AB. A rapid NAD+-linked assay for microsomal uridine diphosphate glucuronyltransferase of rat liver and some observations on substrate specificity of the enzyme. Biochem J 1975;151:131-140. [PubMed: 174550]

21. Tabrett CA, Coughtrie MW. Phenol sulfotransferase 1A1 activity in human liver: kinetic properties, interindividual variation and re-evaluation of the suitability of 4-nitrophenol as a probe substrate. Biochem Pharmacol 2003;66:2089-2097. [PubMed: 14609733]

22. Roy AB. Comparative studies on the liver sulphatases. Biochem J 1958;68:519-528. [PubMed: 13522654]

23. Szczygiel MA, Kusakabe H, Yanagimachi R, Whittingham DG. Intracytoplasmic sperm injection is more efficient than in vitro fertilization for generating mouse embryos from cryopreserved spermatozoa. Biol Reprod 2002;67:1278-1284. [PubMed: 12297546]

24. Ward MA, Burgoyne PS. The effects of deletions of the mouse Y chromosome long arm on sperm function--intracytoplasmic sperm injection (ICSI)-based analysis. Biol Reprod 2006;74:652-658. [PubMed: 16354792]

25. Ajduk A, Yamauchi Y, Ward MA. Sperm chromatin remodeling after intracytoplasmic sperm injection differs from that of in vitro fertilization. Biol Reprod 2006;75:442-451. [PubMed: 16775225] 
26. Cibelli JB, Stice SL, Golueke PJ, Kane JJ, Jerry J, Blackwell C, Ponce de Leon FA, Robl JM. Cloned transgenic calves produced from nonquiescent fetal fibroblasts. Science 1998;280:1256-1258. [PubMed: 9596577]

27. Young LE, Sinclair KD, Wilmut I. Large offspring syndrome in cattle and sheep. Rev Reprod 1998;3:155-163. [PubMed: 9829550]

28. Wakayama T, Perry AC, Zuccotti M, Johnson KR, Yanagimachi R. Full-term development of mice from enucleated oocytes injected with cumulus cell nuclei. Nature 1998;394:369-374. [PubMed: 9690471]

29. Wakayama T, Yanagimachi R. Cloning of male mice from adult tail-tip cells. Nat Genet 1999;22:127128. [PubMed: 10369248]

30. Eggan K, Akutsu H, Loring J, Jackson-Grusby L, Klemm M, Rideout WM 3rd, Yanagimachi R, Jaenisch R. Hybrid vigor, fetal overgrowth, and viability of mice derived by nuclear cloning and tetraploid embryo complementation. Proc Natl Acad Sci U S A 2001;98:6209-6214. [PubMed: 11331774]

31. Young LE, Fairburn HR. Improving the safety of embryo technologies: possible role of genomic imprinting. Theriogenology 2000;53:627-648. [PubMed: 10735055]

32. Porterfield, SP. Endocrine Physiology. St Loius, MO: Mosby Inc; 2001.

33. Tong MH, Jiang H, Liu P, Lawson JA, Brass LF, Song WC. Spontaneous fetal loss caused by placental thrombosis in estrogen sulfotransferase-deficient mice. Nat Med 2005;11:153-159. [PubMed: 15685171]

34. Richard K, Hume R, Kaptein E, Stanley EL, Visser TJ, Coughtrie MW. Sulfation of thyroid hormone and dopamine during human development: ontogeny of phenol sulfotransferases and arylsulfatase in liver, lung, and brain. J Clin Endocrinol Metab 2001;86:2734-2742. [PubMed: 11397879]

35. Collier AC, Ganley NA, Tingle MD, Blumenstein M, Marvin KW, Paxton JW, Mitchell MD, Keelan JA. UDP-glucuronosyltransferase activity, expression and cellular localization in human placenta at term. Biochem Pharmacol 2002;63:409-419. [PubMed: 11853692]

36. Collier AC, Tingle MD, Paxton JW, Mitchell MD, Keelan JA. Metabolizing enzyme localization and activities in the first trimester human placenta: the effect of maternal and gestational age, smoking and alcohol consumption. Hum Reprod 2002;17:2564-2572. [PubMed: 12351530]

37. Ejiri N, Katayama K, Kiyosawa N, Baba Y, Doi K. Microarray analysis on Phase II drug metabolizing enzymes expression in pregnant rats after treatment with pregnenolone-16alpha-carbonitrile or phenobarbital. Exp Mol Pathol 2005;79:272-277. [PubMed: 16223480]

38. Lucier GW, Sonawane BR, McDaniel OS. Glucuronidation and deglucuronidation reactions in hepatic and extrahepatic tissues during perinatal development. Drug Metab Dispos 1977;5:279-287. [PubMed: 17527]

39. Creech Kraft J, Eckhoff C, Kochhar DM, Bochert G, Chahoud I, Nau H. Isotretinoin (13-cis-retinoic acid) metabolism, cis-trans isomerization, glucuronidation, and transfer to the mouse embryo: consequences for teratogenicity. Teratog Carcinog Mutagen 1991;11:21-30. [PubMed: 1677495]

40. Shah HC, McLachlan JA. The fate of diethylstilbestrol in the pregnant mouse. J Pharmacol Exp Ther 1976;197:687-696. [PubMed: 819646]

41. Tzimas G, Collins MD, Nau H. Developmental stage-associated differences in the transplacental distribution of 13-cis- and all-trans-retinoic acid as well as their glucuronides in rats and mice. Toxicol Appl Pharmacol 1995;133:91-101. [PubMed: 7597714]

42. Kuczynski J, Pydzik T, Wenclewski A. Aryl Sulfatase Activity Of The Placenta And Fetal Brain And Liver. Ginekol Pol 1964;35:373-378. [PubMed: 14256127]

43. Zhang Y, Zhang YL, Feng C, Wu YT, Liu AX, Sheng JZ, Cai J, Huang HF. Comparative proteomic analysis of human placenta derived from assisted reproductive technology. Proteomics 2008;8:43444356. [PubMed: 18792929]

44. Miles HL, Hofman PL, Cutfield WS. Fetal origins of adult disease: a paediatric perspective. Rev Endocr Metab Disord 2005;6:261-268. [PubMed: 16311944]

45. Silver RI, Rodriguez R, Chang TS, Gearhart JP. In vitro fertilization is associated with an increased risk of hypospadias. J Urol 1999;161:1954-1957. [PubMed: 10332480] 
46. Wennerholm UB, Bergh C, Hamberger L, Lundin K, Nilsson L, Wikland M, Kallen B. Incidence of congenital malformations in children born after ICSI. Hum Reprod 2000;15:944-948. [PubMed: 10739847] 


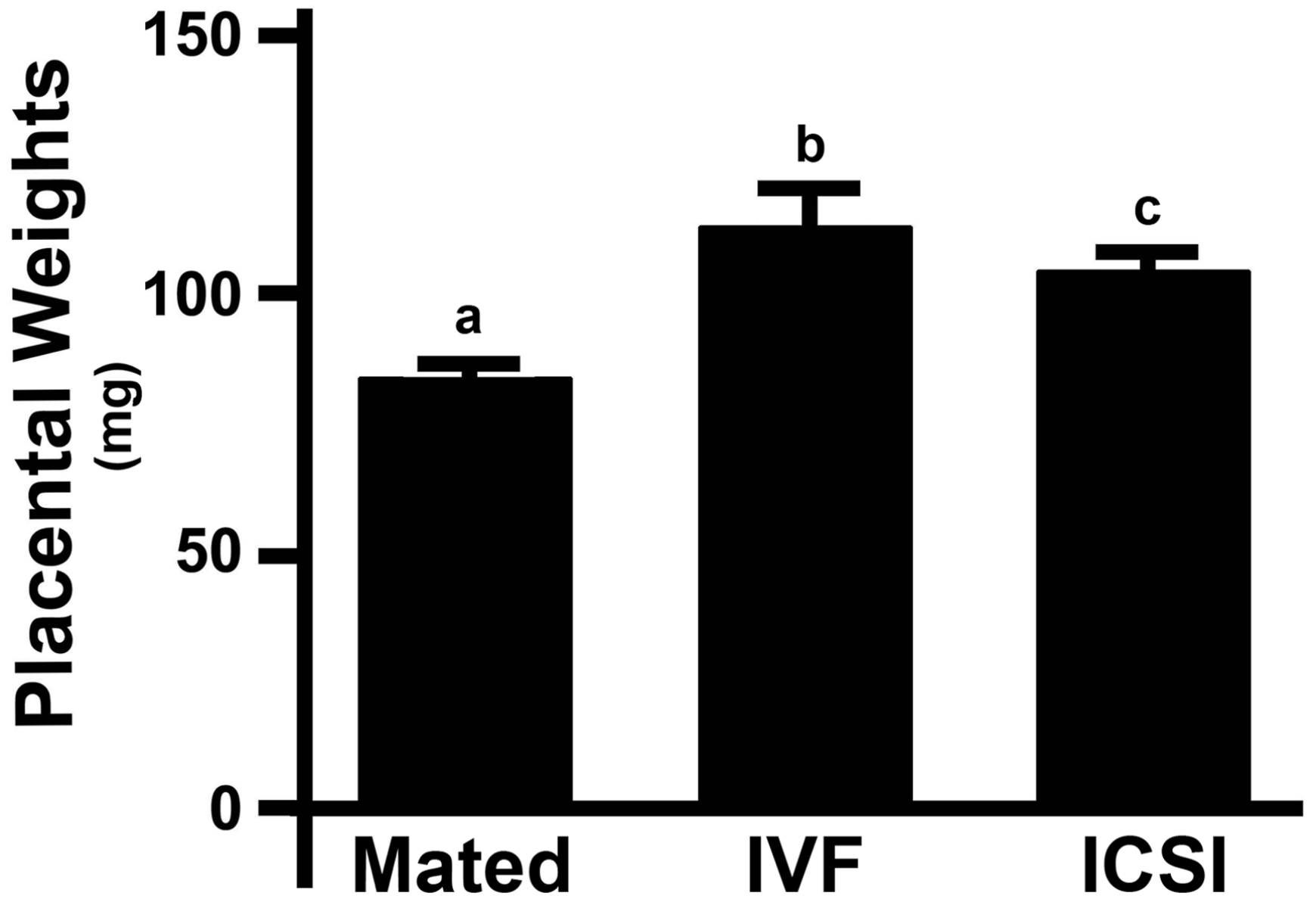

Figure 1. Placental weights in mice impregnated using mating, IVF and ICSI

Each bar represents a mean of 30 placentas \pm SD. Statistical significance: a vs. b, a vs. $c=P$ $<0.001$. 


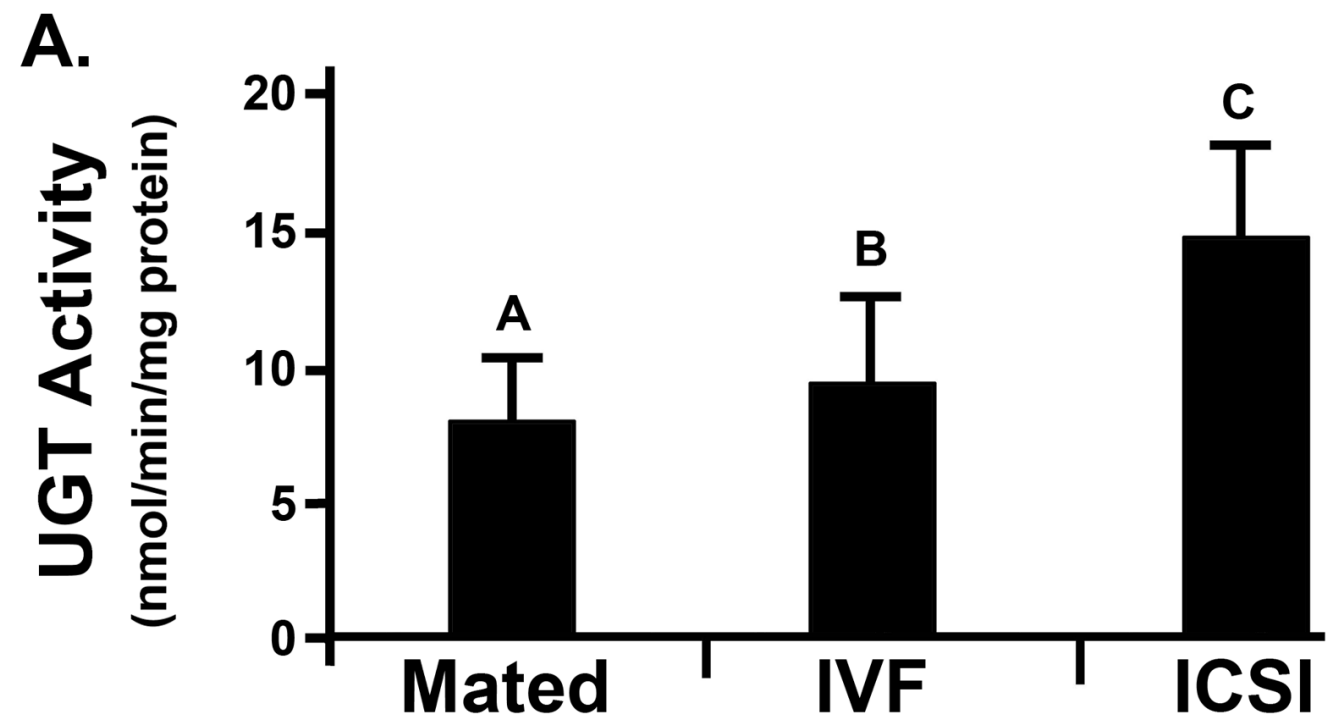

B.
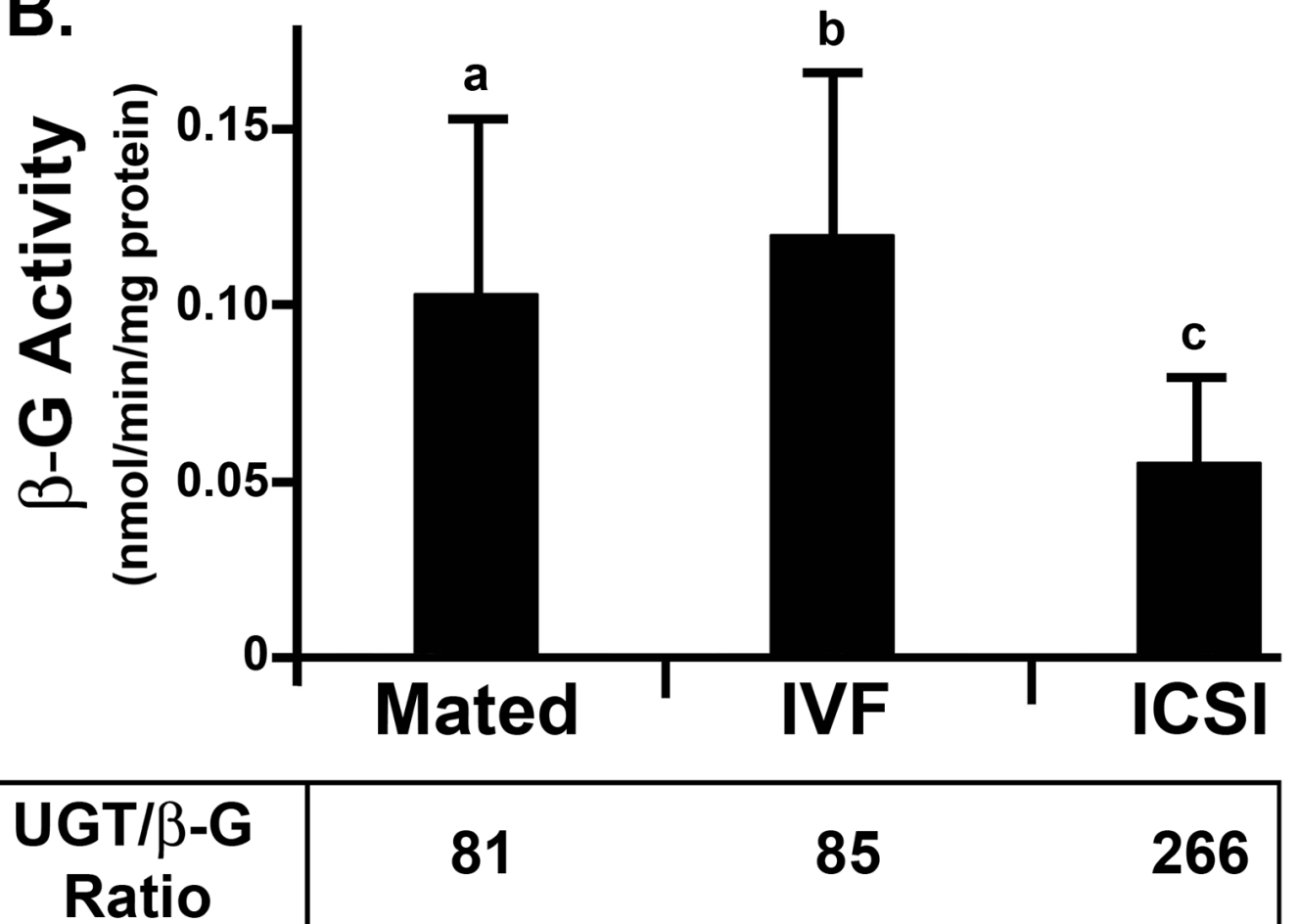

Figure 2. Glucuronidation Activity

A: UGT in placenta; $B: \beta-G$ in placenta. Each bar represents a mean of $n=30$ placentas \pm SD. Statistical significance: A vs. C, B vs. C, A vs. a, B vs. b, C vs. c, a vs. c, b vs. c = P $<0.001$; A vs. $\mathrm{B}=\mathrm{P}<0.05$. For explanation of abbreviations see Table 1 . 

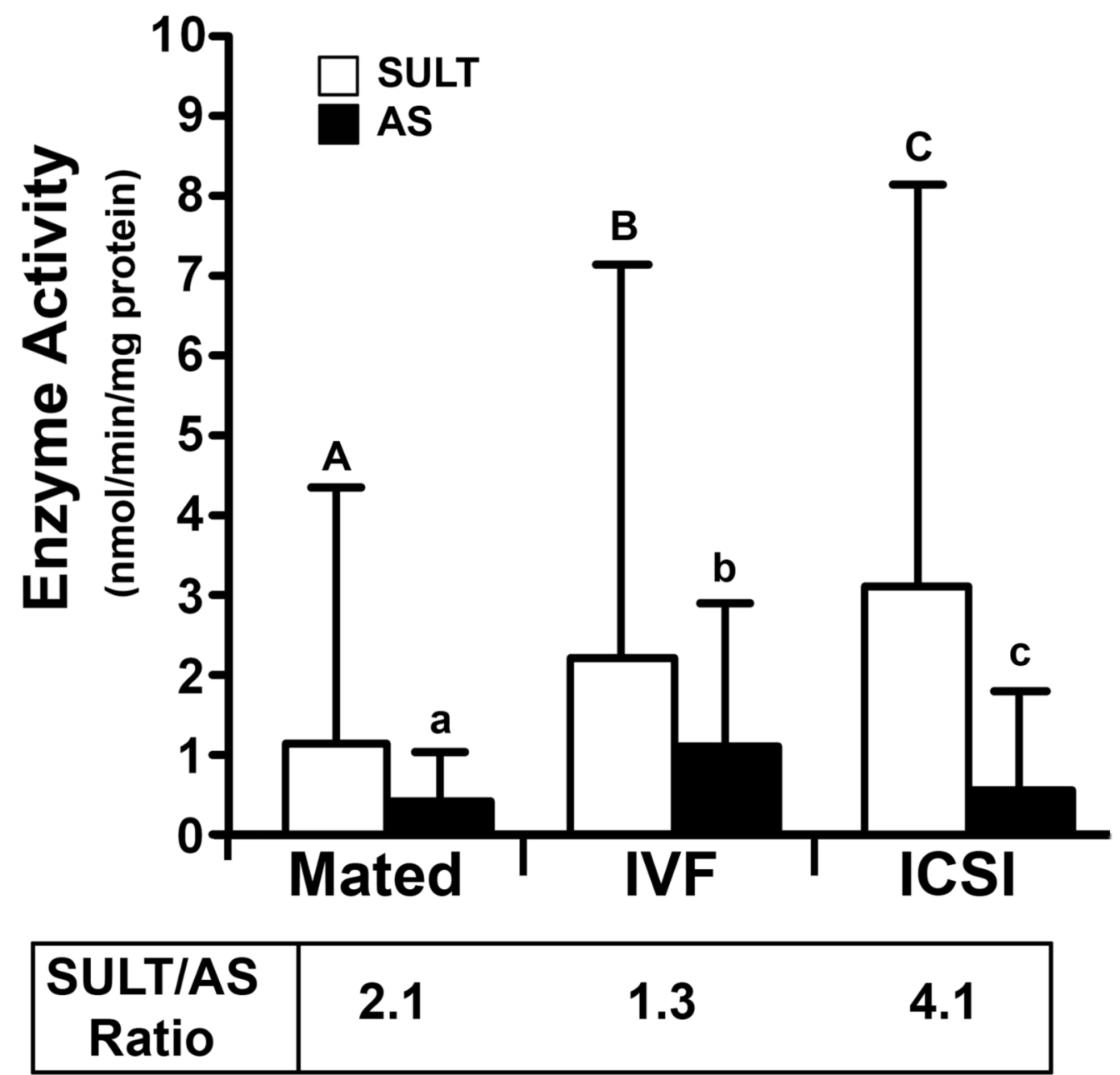

Figure 3. Sulfonation Activity

SULT and AS in placenta. Each bar represents a mean of $n=30$ placentas \pm SD; Statistical significance: $\mathrm{A}$ vs. $\mathrm{C}=\mathrm{P}<0.001 ; \mathrm{C}$ vs. $\mathrm{c}=\mathrm{P}<0.01 ; \mathrm{A}$ vs. $\mathrm{B}, \mathrm{B}$ vs. $\mathrm{C}=\mathrm{P}<0.05$. For explanation of abbreviations see Table 1. 
A.

B.
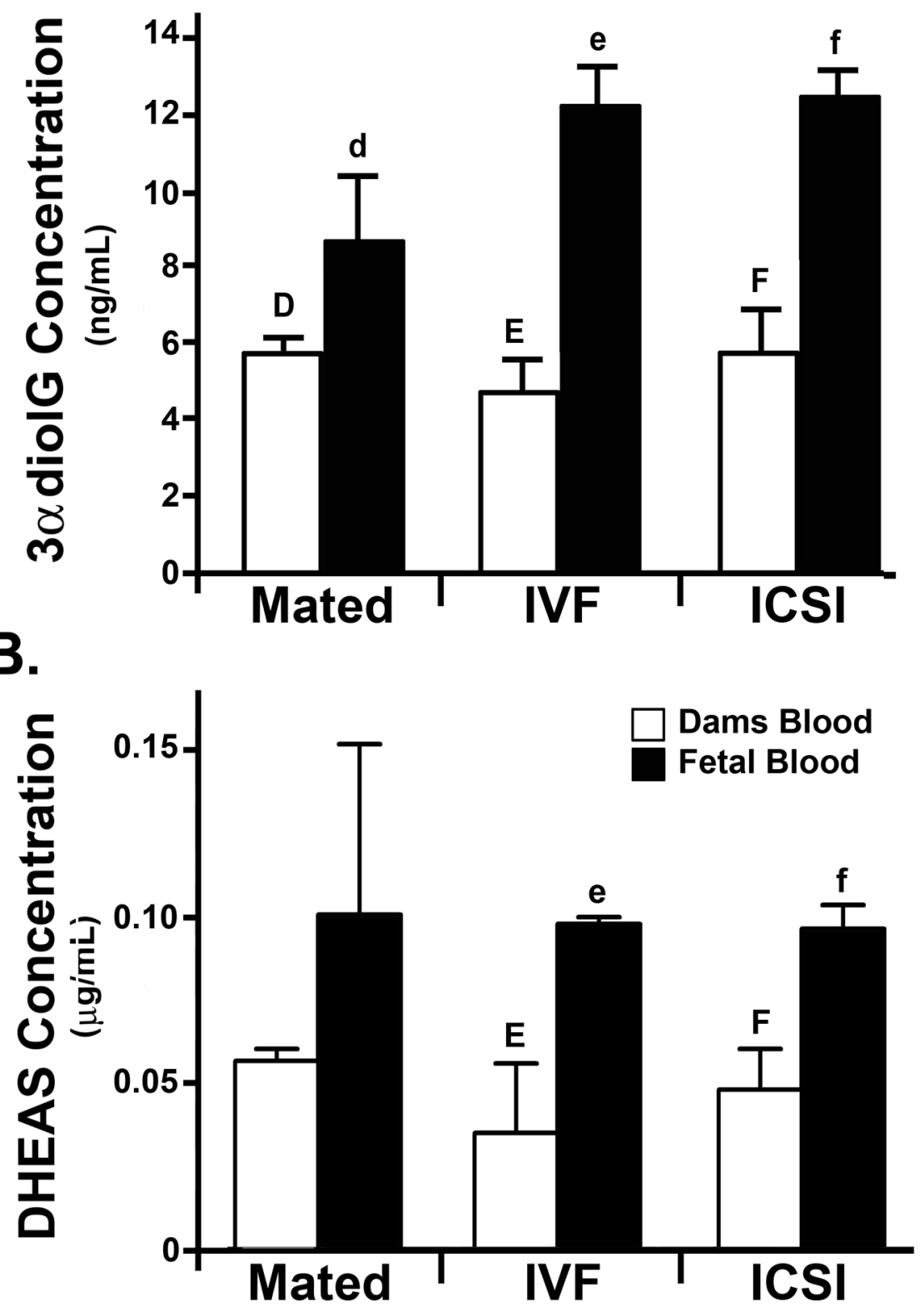

Figure 4. Levels of steroid metabolites in blood plasma from dams and fetuses

A: 3adiolG; B: DHEAS. Each bar represents a mean of $n=3$ samples \pm SD, except for IVF pups, $\mathrm{n}=2 \pm \mathrm{SD}$ ). Statistical significance: $\mathrm{d}$ vs. $\mathrm{f}$, E vs. e, $\mathrm{F}$ vs. $\mathrm{f}=\mathrm{P}<0.05$. For explanation of abbreviations see Table 1 . 


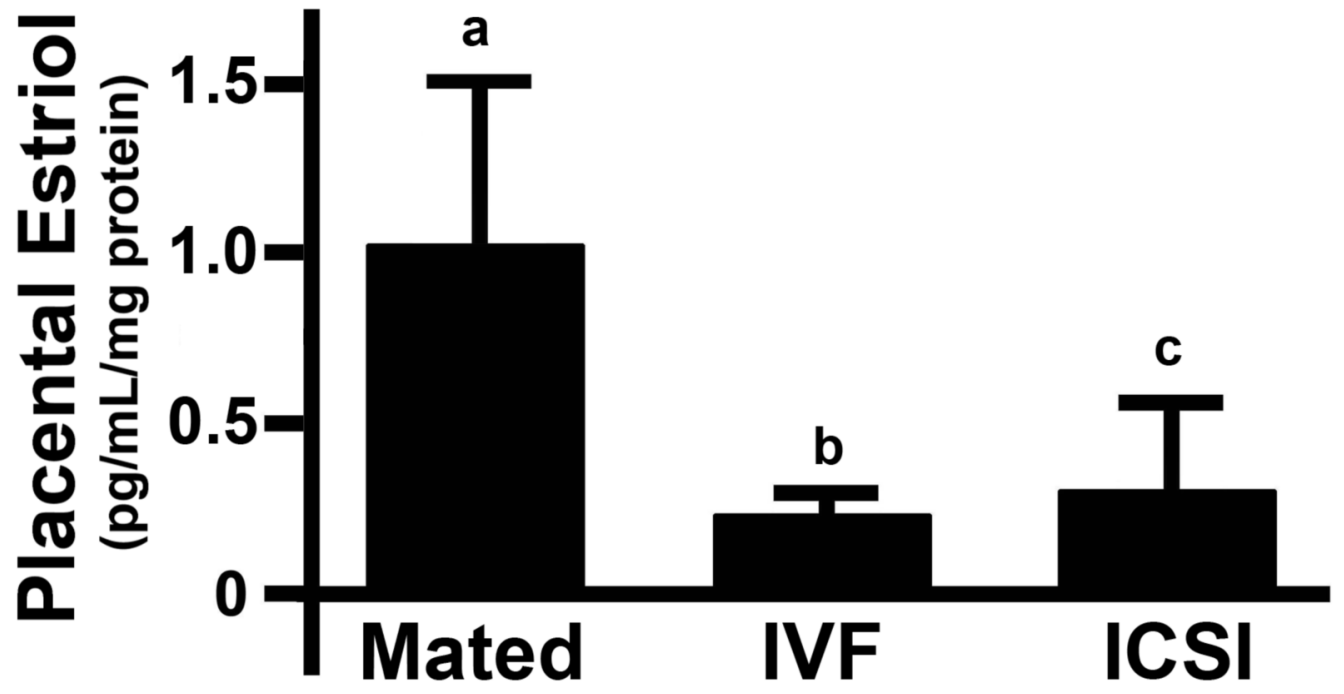

Figure 5. Estriol levels in placentas from mice impregnated by mating, IVF and ICSI Each bar represents a mean of $n=3$ pools (range: $7-15$ placentas per pool) \pm SD. Statistical significance: a vs. $\mathrm{b} \mathrm{P}<0.05$, a vs. $\mathrm{c}=\mathrm{P} 0.09$. 
A.

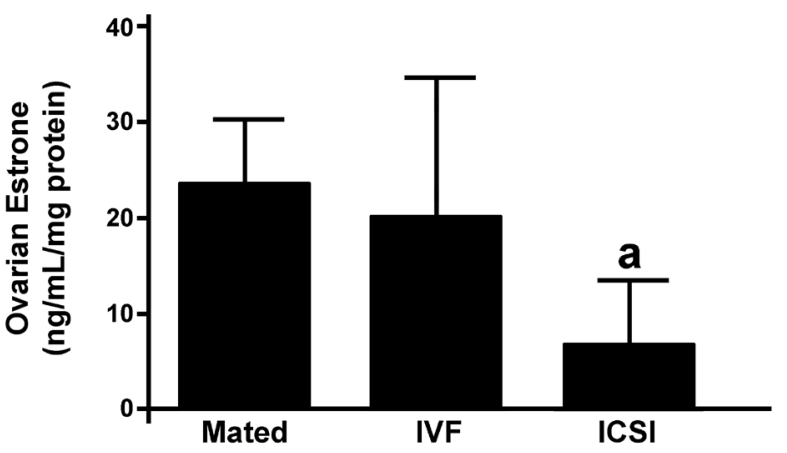

B.

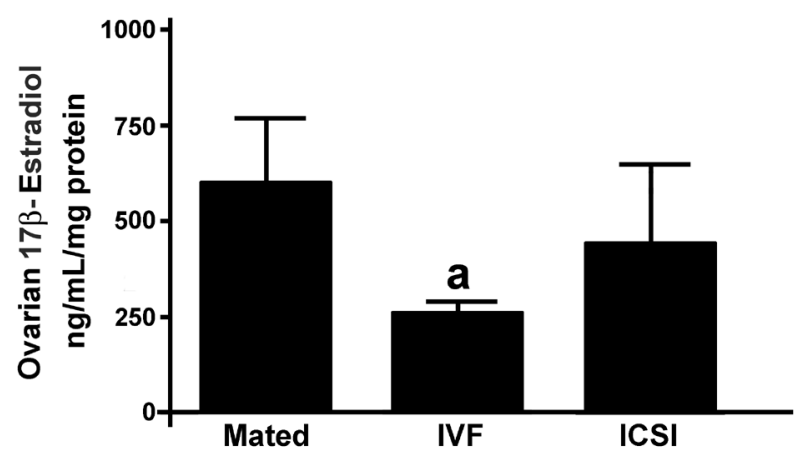

c.

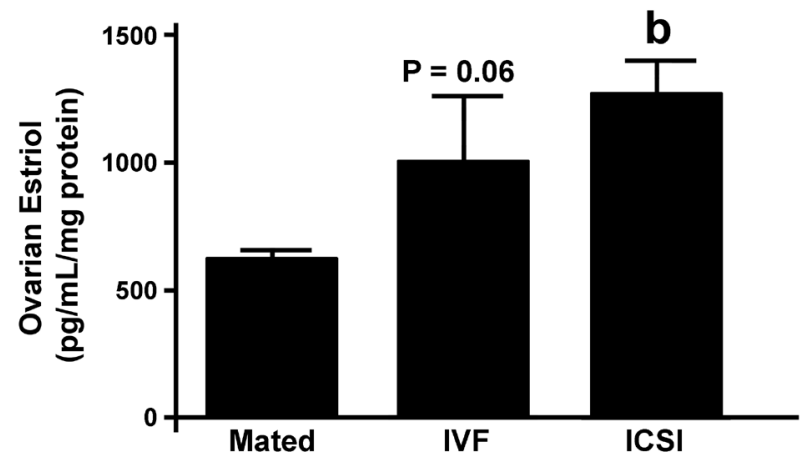

Figure 6. Estrogenic steroid levels in ovaries from mice impregnated by mating, IVF or ICSI Each bar represents a mean of $n=3$ ovaries, \pm SD. Statistical significance: $a=P<0.05$ vs. Mated, $\mathrm{b}=\mathrm{P}<0.001$ vs. Mated. 
Collier et al.

Page 19

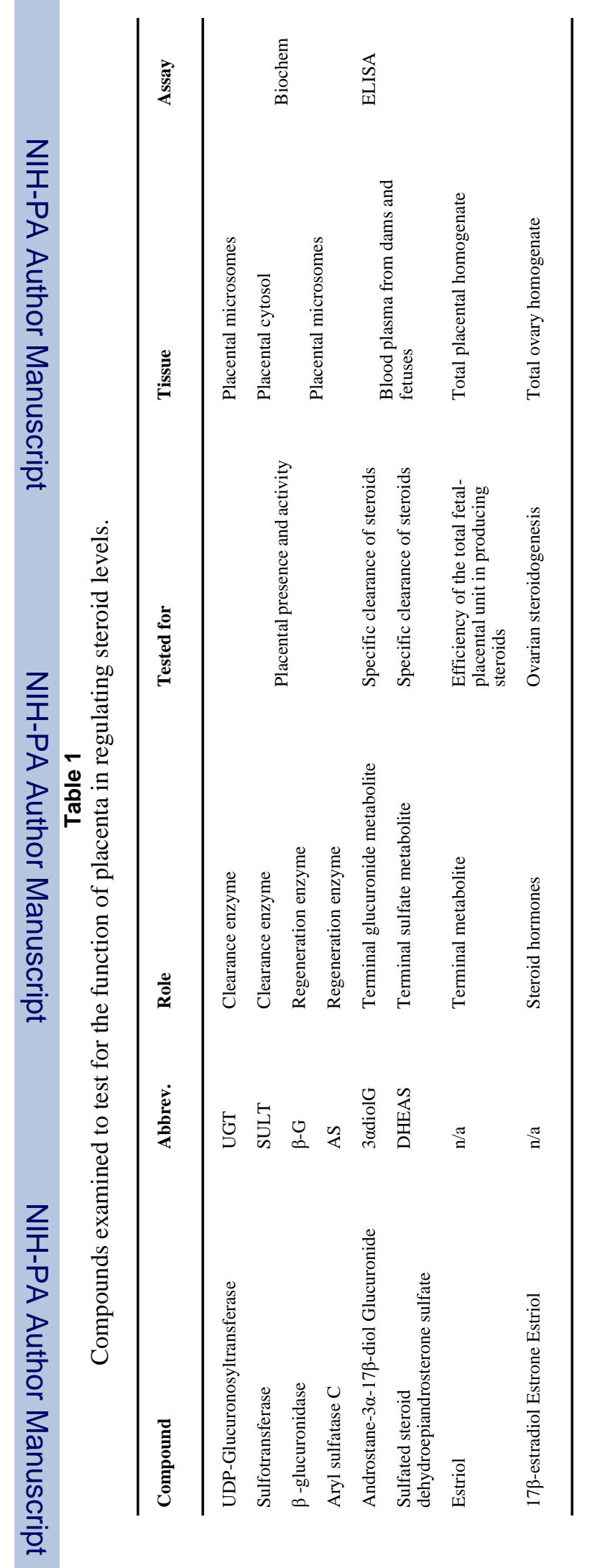

J Steroid Biochem Mol Biol. Author manuscript; available in PMC 2010 August 1. 


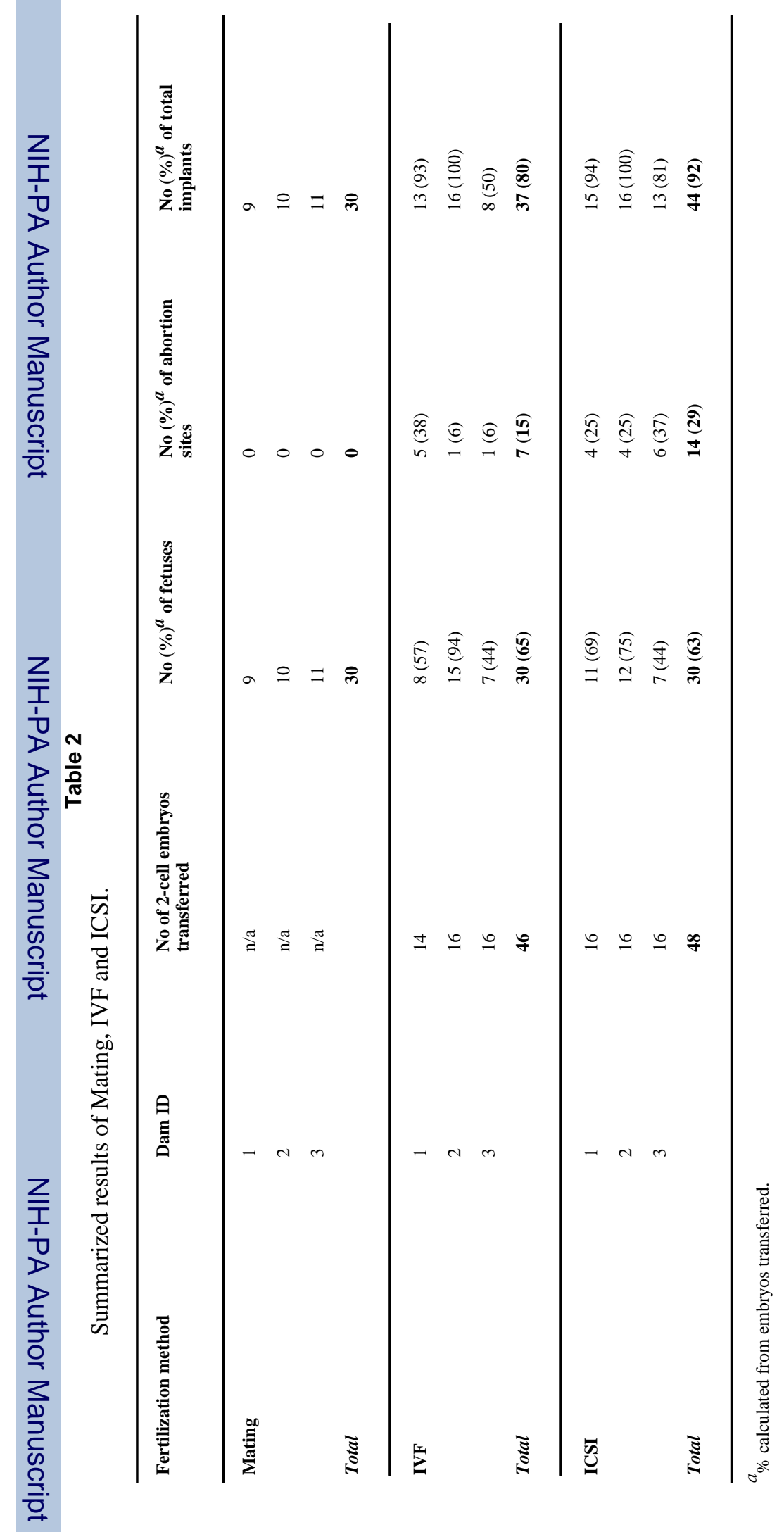

